\section{Abgrenzung und Begriff des neuropathischen Kindes.')}

Von Dr. Ewald Stier, Stabsarzt und Privatdozent für Psychiatrie in Berlin.

M. H.! Das Studium der psychisch-nervösen Störungen des Kindesalters ist noch relativ jung und erst etwa im letzten Jahrzehnt von seiten der Neurologen sowohl wie von seiten der Kinderärzte zunehmend in Angriff genommen worden. Wie groß der Unterschied der jetzigen Einschätzung dieser Störungen gegenüber der der früheren Zeit ist, das erhellt wohl am klarsten, wenn man die Stellungnalıme des jetzigen Direktors der Berliner Universitäts-Kinderklinik, der bei allen Störungen des Kindesalters auf die Mitbeteiligung nervös-funktioneller Abweichungen entscheidenden Wert legt, vergleicht nit dem, was sein Vorgänger in seinem 1906 erschienenen Lehrbuch der Kinderkrankheiten über die gleiche Frage äußert. Heubner teilt in dem genannten Lehrbuch in dem ganz kurz gehaltenen 7. Kapitel die ,sogenannten funktionellen Erkrankungen des Nervensystems" ein in 1. Epilepsie, 2. Hysterie, 3. Enuresis nocturna, und als Anhang zu dem Abschnitt über Hysterie bespricht er als drei gesonderte Krankheitsbilder die Maladie des tics, die Tickrankheit und den Pavor nocturnus; aus dem ganzen großen Gebiet der psychisch-nervösen Störungen ist also überhaupt nur ein ganz kleiner Ausschnitt berücksichtigt worden, und auch dessen Bearbeitung kann, wie schon die 'Teilung in Maladie des tics und Tickrankheit zeigt, nicht als einwandfrei gelten.

Das Beobachtungsmaterial nun, das im letzten Jahrzehnt über die fraglichen Störungen zusammengetragen worden ist kann als recht beträchtlich bezeichnet werden; beteiligt sind bei dieser Arbeit sowohl die Neurologen als auch die Pädiater, und zwar die letzteren meist ausgehend von dem Studium der spasmophilen Diathese, die ersteren von allgemeinen neurologischen oder noch mehr psycho-pathologischen Beobachtungen. Wird man das so angehäufte klinische Material als sehr wertvoll und im allgemeinen geeignet für weiteres Arbeiten bezeichnen dürfen, so kann das Gleiche von der prinzipiellen Stellungnahme zu dem Problem und den Einteilungs versuchen des ganzen Gebiets wohl noch nicht gesagt werden.

Für den Mangel an Uebereinstimmung in dieser Bezielıung seien nur drei Beispiele angeführt.

In dem bekannten Feerschen Lehrbuch der Kinderheilkunde ${ }^{2}$ ) stellt Ibrahim, der den Abschnitt uber funktionelle Nervenkrankheiten bearbeitet hat, die Krampfkrankheiten, die Chorea und dic Psychosen als koordiniert der ,,neuro-psychopathischen Konstitution (hereditäre Neuropathie)" gegeniiber und teilt den letztgenannten Abschnitt ein in "Migräne, Maladie des tics, Wegbleiben, Pavor nocturnus, Nenrasthenie, Phobien ete., Störungen des Trieblcbens (Wandertrieb, Onanie), Hysteric". Er verzichtet also ganz auf eine Unterteilung dieser vom nelırologiseh-psychiatrisehen Gesichtspunkt doch recht ungleichwertigen Störungen. Thiemich dagegen unterscheidet in dem Handbuch der Kinderheilkunde von Pfaundler und Schloss $\operatorname{mann}^{3}$ ) bei den funktionellen Nervenkrankheiten neben den Krampfkrankheiten, der Hysterie und den Psychosen als z wei selbständige Absehnitte die "Ncurasthenie" und die "hereditäre Neuropathie" und bringt damit cinen im Prinzip glücklichen Teilungsversuch, der sich ungefälır mit der nnseres Erachtens besten Teilung in neuropathische und psychopathische Störungen deckt; da Thiemich jedoch $\mathrm{z}$. B. dic Labilität der Stimmungen in den ersten, sonst die neuropathischen Störungen enthaltenden Abschnitt und die Migräne, die Maladie des ticsu. a. in den zweiten, sonst mehr die psychopathischen Storungen enthaltenden Absehnitt bringt, so verwiseht er die Grenze leider wieder sehr, ja er nimmt offenbar auch prinzipicll eine andere Stellung zu dem Versinch einer Teilung ein, als sie vom neurologisehen Standpunkt gewiinseht werden muß, da er ausdriicklich als Neurasthenie die leichteren und als die hereditärc Neuropathie die schwereren Grade des krankhaften Zustandes bezcichnet. Mit dicser Definierung aber drängt er das Moment der Intensität der Krankheitserscheinungen wohl zu sehr und das Moment der mehr oder minder starken

1) Nach einem Vortrag, gehalten im V. f. Inn. M. u. Kindhlk. in Berlin, Pädiatrisehe Abt., am 8. Juni 1914. (Diskussion siehe hier Nr. 16 Berlin, Padiatrische Abt., am 8. Juni 1914. (Diskussion siehe hier Nr. 16
S. 481. $\left.-{ }^{2}\right)(2) 1912$ Jena. S. 469 ff. - 3 ) Leipzig 1910 (2). Unter S. 481.) $-{ }^{2}$ ) (2) 1912 Jena. S. 469 ff. $-{ }^{3}$ ) Leipzig 1910 (2). Unter
dem Titel "Die Krankheiten des Nervensystems im Kindesalter“c ist der fragliche Abschnitt zusammen mit der Bearbeitung der übrigen Nervenkrankheiten des Kindesalters von Pfister, Zappert und Knöpfolmacher auch als einzeln käufliches Buch ersehienen.
Mitbeteiligung der psychisehen Funktionen zu wenig in den Vordergrund. Auch der Veranch des Neurologen Peritz, in seiner Bearbeitung der "Nervenkrankheiten des Kindesalters"1) die fraglichen Storungen einzuteilen in dic 4 Hanptgruppen: Nervosität, Tie, Enuresis, Hysterie, mit der Untertcilung des Tics in Schlaftick, Tactatio capitis noctırna, Salaamkrämpfe und Spasmus nutans kann als eine Lösung der Frage wohl noch nicht angesehen werden, da anch hier allzn Ungleichwertiges koordiniert wird. Das ist mn so mehr zul hedanern, als gerade Peritz in dem Abschnitt uber Nervositat dem Wesen der hierler gehörigen krankhaften Zustände wohl am besten von allen gerecht wird.

Dic Gründe, weshalb es bisher noch so garnicht gelungen ist, über die Abgrenzung und Einteilung der genannten Krankheitszustände $\mathrm{zu}$ einer Uebereinstimmung der Auffassungen zu gelangen, liegen nun unseres Erachtens darin, daß so gut wie alle Autoren bei den Einteilungsversuchen noch zu sehr festhaften an dem altgewohnten Begriff der Krankheit und bei dem Versuch, die fraghichen Störungen zu Krankhıiten zusammenzufassen allzu leicht in Gefahr geraten, den Tatsachen Gewalt anzutun. Alle Krankheiten, d. h. Störungen der Funktionen, die eine gewisse Konstanz in den Symptomen und der Symptomgruppierung, in Verlauf, in der Beeinflussungsmöglichkeit und, wenn es gut geht, auch in der nachweisbaren Vcrursachung und der anatomischen Grundlage aufweisen, haben aber offenbar zur Voraus. setzung, daß der betroffene Organismus in sich fertig ist; nur wenn die fraglichen Organismen anatomisch und funktionell im Prinzip sich gleichen, werden auch auf gleiche Ursachen hin gleiclic oder vergleichbare, bis zu gewissen Graden einheitliche Krankheitssymptome auftreten, die eine Zusammenfassung zu einem ,Krankheitsbild" möglich machen. Bei dem noch in der Entwicklung befindlichen Organisnus dagegen werden, abgesehen von einem etwaigen Stillstand der Entwicklung, die gleichen Schädigungen in jedem Stadium der Entwicklung vorschiedcnarige Störungen hervorrufen, die eine Zusammenfassung zu cinhcitlichell Krankheitsbildern erschweren oder unmöglich machen.

Auf dem Gebiet der ,,somatischen" Störungen - wenn es einmal gestattet ist, diese nieht ganz treffende Bezeichnung zum Unterschied gegenüber den psychisch-nervösen Störungen $\mathrm{zu}$ verwenden - ist eine Ungrcnzung von echten Krankheitsbildern denngemä 3 schon im Kindesalter möglich; denn iu rein anatomischer und auch in funktioneller Beziehung ist ${ }^{*}$ auf diesem Gebiete das Kind gleich nach der Geburt fortig und zeigt nur bezüglich der Ernährungsfunktionen für die erste Zeit noch einige Besonderheiten von wesentlicher Bedeutung. Es gibt daher sehr wohl schon beim kleinen Kinde echte somatische Krankheiten in der oben umgrenzten Begriffsfassung, und der Unterschied zwischen diesen Krankheiten des Kindes und denen des Erwachsenen ist mehr quantitativer als qualitativer Natur, d. l. es handelt sich bei diesem Unterschiede vor allem um ein ,häufiger oder seltcner“, um ein ,,mehr oder weniger", und so gut wie alle diese Krankheiten des Erwachsenen können auch beim Kinde und so gut wie alle Krankheiten des Kindesalters können auch bein Erwachscnen vorkonımen, abgesehen auch hier voll den Ernährungsstörungen des Säuglingsalters, die prinzipielle Unterschiede gegenüber den Ernährungsstörungen des Ërwachsenen zcigen.

Auf dem Gebiet der psychisch-nervöscn Funktionen dagegen liegen die Verhältnisse anders. In psychischer Beziehung ist das Kind bei der Geburt noch völlig unfertig. Hier sind nur die Grundlagen für die Reaktions möglich. keiten durch den anatomischen Bau in Prinzip fixiert. Die Entwicklung und Ausbildung der Funktionen geschieht erst unter dem Einfluß der Lebensreize im Laufe der Kindheit, in der Zeit voll der Geburt bis zur Pubertät und darüber hinaus. Ohne eineı allzu sehr hinkenden Vergleich auszusprechen, kalın man daher sagen, daß dic Kindlıeit für die psychische Entwicklung ungefähr das Glciche bedeutet, wie die Zeit des intrauterinen Lebens für die somatische Entwicklung. Als kurze Beispiele dafür, daß in der ersten Lebenszeit das Großhirn überhaupt funktionell noch nicht in Betracht kommt, sei nur an den bekannten großhirulosen Säugling der Heu bner. schen Klinik erinnert, der sich in der kurzen Zeit seines Lebens

1) Berlin 1912, $491 \mathrm{~S}$ 
genau so benahm wie ein normal gebildeter Säugling, und an die weitcre Erfahrung, da Bhitungen oder sonstige bei dem Geburtsakt anftretende Läsionen des Großhirns in der ersten Zcit keinerlei Funktionsstörungen crkemnen lassen und erst, wenn das Kind anfängt, bewul3te Greifbewegnngen oder Stehund Gehversuchc zu machen, die oft reeht beträchtlichen Störungen manifest werden.

Fertig dagegen sind auf dem Gebiet der nervösen Funktionen sehon bei der Geburt der einfache Reflexmechanis. mus, der die Vercinheitlichnng der Funktionen der verschiedenen Organc ermöglieht, und ferner die crsten, etwas lıöheren nnd komplizierteren Funktionen, dic artkonstant sind und als Triebe und Instinkte, als Strebungen und Wollungen nit den hegleitenden einfachen Gefïhlen für die Erhaltung des Lebens unerläßlich sind; ich erinnere nur an das Sclireien bei Hunger und später bei Beschmutzung, und im weiteren Leben an die Abwehr der schädlichon und unangenehmon Beeinflussungen von außen und die Hinwendung zu lustbctonten, für das Leben erforderlichen Reizen. Auf diese elementaren Funktionen, von denen die letzteren schnell eine Weiterentwickhıng zeigen, setzt sich dann allmählich erst das durch individuelle Lcbens. erfahrungen cntwickeltc und ansgestaltcte bewußte Verstandesleben als letztes Stockwerk auf. Die Zeitfolge dieser Stadien der ontogenetischen Lintwicklung entspricht, wenigstens in großen Ziigen, der phylogenetischen Entwicklung. Denn auch in der aufsteigenden Tierreihe sehen wir, als Weiterentwicklung der unmittellbaren Reizbarkeit des Protoplasmas, die in den von Jacques Loeb besonders studierten Tropismen ihren höchsten Ausdruck findet, der Entstehung von Ganglienzellen nnd Fasern entsprechend, zunächst den Mechanismus der Reflexe auftreten, der der Vereinheitlichung der sich differenzierenden Organsysteme dient; dann erst, mit der Entwicklung oincs rndimentären und später nmfangreichen Gehirns, sehen wir kompliziertere Leistungen zu Tage treten, die wir als Reflexe höherer Art bezeichnen kömmen und die sich dadureh auszeichnen, $\mathrm{da} B$ auf bestimmte endogene oder exogene Reize Reaktionen auftreten, dic eine gewisse Anpassung an die jeweilige Situation zeigen, in letzter Linie aber noch artkonstant sind und den Zwecken der Erhaltung und Behauptung des Indivi. duums und der Art dienen; es sind das die oft hoch komplizierten Trieb- und Instinkthandlungen der Tiere bei der Nahrungsbeschaffung, Begattung, Brutpflege etc., die zweifellos schon von einer als psychisch zu bezeichnenden Reaktion des gesamten Individuums begleitet sind, dic wir Gefïlıle zu ncnnen berechtigt sind. Der Entwicklıng einer Großhirnrinde und der höheren Sinne und ihrer Zontren parallel treten dann die höchsten Leistungen des Nervensystems auf, die eine nicht mehr für die Art, sondern schon fïr das Individuum charakteristische Reaktion auf die Außenwelt und die Verwertung individueller Lebenserfahrungen ermöglichen und deren höchste Entfaltung wir in den intellektuellen Funktionen des erwachscnen, rcifen Menschen vor uns sehen.

Im Leben des erwachsenen Mensehen durchdringen diese verschiedenen Funktionen sich in so inniger Weise, da $B$ eine Trennung nur in Ausnahmefällen und auch dann nur zum Teil möglich ist. Boim Kinde aber können wir, da die Entwicklung der einzelnen Stadien zeitlich bis zu gewissen Grade nach einander erfolgt, olme Zwang eine solche Drciteilung wenigstens prinzipiell vornehmon. Und diese Teilung ist es, die mir wesentlich erscheint für das Auseinanderhalten bzw. die Zusammenfassung der einzelnen Funktionsstörungen nach einheitlichen Grundsätzen. Wir erhalten dam die folgende Teilung:

1. neuropathische Störungen = Störungen des phylo. genetisch ältesten, bei der Geburt fast oder ganz fertigen elementaren Reflexmechanismus im weitesten Sinne des Wortes;

2. psychopathische störungen = Störngen des phylogenetisch jüngeren, in der Kindlieitscntwicklung früh auftretenden, artkonstanten Trieb- und Instinktlebens;

3. in tellektnelle Störmngen = Störungen des phylogenetisch jüngsten, in der Kindheitsentwicklung erst später auftrctenden und länger einer Entwickhıng zugängigen, individuell in weitem Maße differenten Verstandeslebens.

Diese 3, wie schon erwähnt, stockwerkartig sich entwickelnden Funktionsgruppen zeigen weitcrhin das gemeinsame Merk- mal, daß die Lebhaftigkeit der zuerst fertigen Funktionen dureh die Entwicklung der höheren Stufen gehemmt und reguliert wird, da $\beta$ also die anfangs sehr lebhaften primären Reflexe, ebenso wie die beim Kinde lebhaften und das Handeln anfangs allein regnlierenden triebhaften Reaktionen an Intensität abnehmen, je weiter die höheren Stufen des Seelenlebens sich entwickeln, bis schließlich die wohl abgewogenen, verstandesmäßig regulierten Willenshandlungen des reifen Mannes als höchste scelische Leistungen resultieren. Die krankhaften Störungen manifostieren sich dann immer als konstitutionellc Anomalien, also als Reaktionen eines in der An. lage abweichenden Organismus anf die Lebensreize, und zwar entweder, wie beim einfachen Reflexmechanismus allein, in einer Perseveration oder Uebertreibung der frül. infantilen physiologischen Uebererregbarkeit, oder, wie beim Trieb- und Instinktleben, in einer Stcigerung oder Abschwächung der intentionalen und emotionalen Elemente des Seelenlebens.

Eine ausführliche Bearbeitung dor letztgenannten, psychopathischen Störungen des Kindesalters an der Hand von 65 meist auf jahrelanger Beobachtung fußenden Krankengeschichten wird demnächst erseheinen.

Betrachten wir heute die erste der genannten Gruppen, dic neuropathischen Störungen, in kurzer Zusammenfassung, so finden wir als gemeinsames dominierendes Merkmal die krankhafte Steigerung der elementaren reflektorischen Erregbarkeit, die das Band bildet, das eine Fülle bekannter nervöscr Störungen des Kindes zu einer einheitlichen Gruppe zusammenhält, und die in ganz nahon Beziehungen zu dem Leben und der Reizbarkeit der Zellen und Gewebe selbst steht.

a) Unmittelbar aus der reflektorischen Uebererregbarkeit ableitbar sind zunächst diejenigen Störungen, die auf einer Herabsetzung der allgemeinen Reizschwelle, auf einer Steigerung der Intensität der normalen Reaktion oder auf einer übergroßen Ausstrahlung der Erregung auf Gebiete beruhen, die normaler Weise durch die fraglichen Reize nicht erregt werden. $\mathrm{Zu}$ den letzteren Störungen gehört vor allem die schon beim kleinen Säugling oft nachweisbare gesteigerte Schreckhaftigkeit, die darin ihren Ausdruck findet, daß schon auf mittelstarke Reize der verschiedenen Sinnesgebiete - grelles Licht, lauter Schall, Erschütterung, Berührung, Anblasen etc. - ein Zusammenzucken größerer Gebiete oder der ganzen Körpermuskulatur erfolgt.

Auf der gleichen Ucbererregbarkeit, aber mehr in Sinne einer gesteigerten Ansprechbarkeit aller Sinnesgebiete für leichte oder sehr leichte Reize, beruht offenbar auch die Muskel. unruhe, die wir bei den neuropathischen Säuglingen manchmal, bei den etwas älteren Kindern so häufig beobachten. Der Gegensatz zwischen dem ruhig und friedlich daliegenden normalen Säugling einerseits, der beim Herantreten von Personen, bcim Anblasen, bei der Annäherung von Licht, bei leichten Geräuschen, bei dem Nahen der Flasche etc. freundlich lächelt, und dem nenropathischen Säugling anderseits, der sofort unruhig die Glieder bewegt, den Kopf hebt, in die Luft greift, sich umsieht, den Saugpfropfen der Flasche zu schnell erfaßt, danebenfährt, hastig trinkt, bei jedem Sinnesreiz die Flasohe wieder losläßt und von neuem schlecht erfaßt und auch nach anderen Gegenständen schnell, aber schlecht und ohne Energie zugreift, ist so evident und charakteristisch, $\mathrm{da} \beta$ er die Diagnose meist sofort erkennen läßt. Beim älteren Kinde sehen wir die gleiche Unruhe in etwas anderer Form; hier beteiligen sich vor allem die Hände, der Kopf und etwa auch die Beine an der Unruhe; die Kinder fassen alles an, können nicht stillsitzen und nicht stillstehen, rücken hin und her, stehen auf einem FuB, nehmen den $F u B$ in die Hand und bilden durch diese ewige Unruhe eine Qual für Eltern und Erzieher. Hand in Hand geht mit dieser übermäßigen Beweglichkeit der ganzen Muskulatur fast stets bei diesen Kindern eine gesteigerte Er müdbarkeit und Unfälnigkeit, sich zu konzentrieren, wenn auch ganz konstante Beziehungen zwischen der gesteigerten Ermüdbarkeit und der Muskelunruhe nicht bestehen.

Eine weitere Folge der Uebererregbarkeit der Muskulatur schon durch schwache Sinnesreize und überhaupt der Herab. setzung der Reizschwelle sind die Schlafstörungen, die sich in erschwertem Einschlafen, unruhigem Weiterschlafen und 
zu leichtem Erwachen manifestieren. Hicr wirken cben die Reizungen durch schwaclıes Licht, durclı leichte Geräusche, durch schlechte Lagerung, durch Druck der Decke, durch zu große oder zu geringe Erwärmung cinzelner Körperteilc schon in solchem Maße erregend, daß die Gewinnung oder Erhaltung einer normalen Schlaftiefe gestört ist. Die sonstigen Sclılafstörungen des Kindesalters, das Sprechen und Umlıcrwandeln im Schlaf und der eclitc Pavor, sind an sich kompliziertere Störungen, die aber immer auf dem glcichen Boden der ncuropathischen Schlafstörungen erwachsen und prinzipiell mit der Epilepsie nichts zu tun labell.

Der Schreckhaftigkeit nahe verwandt sind auch die aus der neuropathischen Uebererregbarkeit unmittelbar hervorwachsenden Kinderk onvulsionen, die besonders von den Kinderärzten in den letzten $10 \mathrm{Jahren}$ genauer studiert sind und die in dem Begriff der Spasmopliilie einen zusammenfassenden Ausdruck gefunden haben. Hier erfolgt auf Reize, deren Natur uns im einzclnen nichıt immer klar vor Augen liegt, eine plötzliche, aber länger als bei der einfachen Sclıreckhaftigkeit anhaltende krampfhafte Zusammenziehung einzelner Muskclgebiete oder der gesamten Muskulatur. Für die Uebererregbarkeit stellen diese Störungen insofern geradezu den Urtyp dar, als wir die Uebererregbarkeit an den peripherischen Nerven durclı Anwendung mechanischer oder elektrischer Reize unmittelbar feststellen und messen können und seit den Arbeiten von Thiemich sogar für die KOcZ eine im Prinzip allgemein anerkannte Grenzc kennen, von der an cinc Uebercregbarkeit als krankhaft bezeichnet werden mu $B$ und auel bei dem Fehlen eklatanter Erscheinungen zur Diagnose der Spasmophilie ausreicht. Im einzelnen auf diese Fragen einzugelien, ist hicr nicht der Ort.

Wichtig ist weiterhin, daß die Uebererregbarkeit nicht nur im Gebiet des zentralen, besonders des spinalen, sondern auch im Gebiet des glciclifalls pliylogenetisch alten, vegeta. tiven Nervensystems sich zeigt. Hier ist es die Steigerung der Vasomotilität, die auf leichte Reize verschiedcuer Art schon ein Erröten, ein Erblassen auslöst und bald in einfacher Ueberempfindlichkeit gegen Kälte und Hitze, bald in krampfhafter, umgrenzter Kontraktion der Blutgefäße - Totenfinger Pseudo-Raynaudsche Krankheit - an den Fingern sich zeigt oder dic letzte Ursache für das erleichterte Auftreten von Ohnmachten und Angstzuständen bildet; in anderen Fällen sind cs mchr die Störungen der sekretorisehen Funktionen vermehrter Schweißausbruch, nervöse Polyurie, Ungleiclınäßigkeiten in der Absonderung des Magen- und Darmsaftes die zu Störungen der Ernährung Veranlassung geben. Dic Ueberempfindlichkeit des vegetativen Nervensystcms führt ferner im Gebiet des kardiovaskulärcn Systcms zu Herzklopfen, Pulsbeschleunigung und Pulsarythmien, im Gebict der Atmungsorgane zu krampfhaften Zusammenziehungen der Muskulatur der kleinsten Bronchien und damit zu asthmatischen Erscheinungen ${ }^{1}$,) im Gebiet der Verdallungsorgane zu Durchfällen, Erbrechen und den Symptomen des Pylorospasmus.

Gleichzeitige Erregbarkeitsstcigerung in bciden Gcbieten, sowohl im zentralen als im vegetativen Nervensystcm, müssen wir schließlich als Hauptursache anselıen für die im Kindesalter so unendlich wichtigen Störungen der Enuresis und der verfrühten Erektionen der ganz kleinen Knaben. Während normalerweise der Reflexmcclianismus der Blasen. entlcerung beim Säugling und kleinen Kinde rein automatisch dann eintritt, wenn die Blase bis zu einem gewissen Grade gefüllt ist und die Blasenwände so eine bestimmtc Spannung erfahren, tritt bei Uebercmpfindlichkeit der nervösen Elenientc der Blasenwände der glciche Reflexmechanismus frülicr und damit häufiger cin. Die Beherrschung dieses Mechanismus und die willkürlichc Hinauszögerung der Blasenentleerung, die mit zunelımender Entwicklung der Großhirnfunktionen von dem normalen Kinde früh crlernt wird, wird vom neuropathischen Kinde erst später erlernt, da eben durch die Steigerung der Erregbarkeit nicht nur häufiger, sondern auch mit größcrer Gewalt und Schnelligkeit der reflektorische Mcchanismus in

1) Auf die innigen Beziehungen zwisehen Spasmophilie und asthmatischen Erseheinungen ist neuerdings von $K a s s o w i t z$, Lederer, Rietsehel und Rosenstern hingewiesen worden.
Aktion tritt. Der gehäufte und imperatorische Harndrang ist also zur Feststellung des zugrunde liegenden krankhaften Zustandes im ganzen wichtiger als die Tatsache des Finnässens bei Tage oder ihre abgeschwächte und schon gemilderte Form des nächtlichen Einnässens, bci der die willkürliele Hemmung bei Tage zwar schon gelingt, im Schlaf aber noch reflektorisch erfolgt. Dieser gehäufte Harndrang erlcichtcrt auch die Unterscheidung von der anderen großen Gruppe von einnässenden Kindern, bei denen die Bcherrschung des Reflexmechanismus deshalb verspätet gelingt, weil wegen des bestehonden Scliw achsinns die höheren Verstandesf unktionen und damit die Fähigkeit zur willkürlichen Belıerrschung des Harndranges überhaupt verspätet sich cntwickeln. Die hochinteressanten neueren Forschungen über Störungen in der Konfiguration der Lenden- oder Sakralwirbel und Störungen der Sensibilität, die einen noclı direkteren Schlıß auf das Bestehen einer Myelodysplasie bei den bettnässenden Kindern zulassen, sind natürlich nicht geeignet, die prinzipiellc Auffassung der Störungen zu ändern; sic können liöchstens uns das Verständnis dafür erleichtern, wie wir den Mangel an $\mathrm{Hcm}$ mung durch zentrale Beeinflussung uns auch anatomisch vorstellen können. Etwas wirklich Beweisendes haben ja alle diese neueren Untersuchungen natürlich überhaupt noch nicht, solange cs an anatomischen Präparaten des Rückenmarks mit nachweisbaren $M \cdot B b i l d u n g e n$ und Veränderungen fehlt, die wir mit dem Symptom des Einnässens in Verbindung bringen können.

Daß auch die Erektionen der kleinen Knaben in den ersten drci Lebensjahren etwa den unmittelbaren Ausdruck der Uebcrerregbarkeit der fraglichen Zentren oder peripherischen Nervenendorgane darstellen, bcdarf keiner Begründung. Sic stellen offenbar ein fast physiologisches Symptom der frül. kindlichen nervöscn Ucbererregbarkeit dar, verscliwinden fast stets mit dem 3.-4. Lebensjahre und bilden nur dadurclı eine Gefahr, da $B$ die Kinder zur Bescitigung des eigcnartigen Reizes manuelle Friktionen dazutın und so zur Onanie gelangen können. Praktisch halte ich diese Gefahr jedoch für selır gering, glaube vielmehr auf Grund meiner Erfahrungen, daß die früh zur Onanie kommenden Kinder generell Ps ychopatlien sind und die Störungen hier nicht auf einfachc reflektorische Uebererregbarkeit zurückgefülırt werden können.

b) Als eine zweite Hauptgruppe der neuropatlischen Störungen kommen diejenigen Symptome in Betracht, be denen weniger der motorische Enderfolg der gesteigerten reflektorischen Erregbarkeit in Vordergrunde steht als vielmelır das verfrühte Auftreten des Unlustgefühls. Während normalcr Weise alle schwachen Reize lcicht positiv, also lustbctont sind, bei zunclimender Intensität des Reizes dann zunäclıst das Lustgefühl nachläßt und erst bei relativ starkem Reize cin Unlustgefülıl sich einstellt, schen wir bei den übererregbaren Kindern und Erwachsenen dies Unlustgefühl niclit nur an normaler Stelle besonders stark, sondern auch zu frülı bci Reizen von nur geringer Intensität, also dann eintrcten, wenn bei anderen Mcnschen ein Unlustgefülll noch nicht anklingt. Mit dicsen zu früh einsetzenden Unlustgcfühlen ist dann meist, aber nicht immer, auch eine gesteigcrte motorische Reaktion in dem zugehörigen motorischen Gebiet verbunden.

Als Bcispiele für diese Art von Störungen seien nur erwähnt die starken Unlustgefühle vieler Kinder bei Geruchs. und Geschmacksreizen, dic bei anderen Kindern kcin Mißbchagen oder gar Wollgefallen auslösen und dic besonders dadurch, daß sie leicht reflcktorisch zu Erbrechen führen, oft ein sclıweres Hindernis für die Ernährung darstcllen. Weniger häufig ist die Lichtscheu der kleinen Neuropathen; schr wichtig aber wieder die Ucbercmpfinrllichkeit gegen Gerä usch c. Denn da erst mit zunehmender Bedcutung der Sprache auch dic Hörvorstellungen eine größere Rolle für das seelische Leben gcwinnen und wir dementsprechend einc Ueberempfindlichkcit gegen Geräusche im allgemeincn erst bei Erwachsenen eintreten sehen, muß das Auftreien solcher Ucberempfindlichkeit schon bei Kindern vor oder in der ersten Schulzeit als cin Zeichen ciner beträchlichcren Entwicklungsstörung angesehen und darum ernster bewcrtet werden. Claraktcristisch ist dabei, daß stets nur die von anderen, nicht die von dem Kinde sclbst 
hervorgerufenen Geräusche unangenchm empfunden wcrden; das Lustgefühl, das mit jeder Aktivität verbunden ist, ist offenbar für das Kind so groß, daß die Freude an dell cigenen, mit Lärın verbundencn Leistuıgen inımer auch dic sonst etwa vorhandene Ueberempfindlichkeit überwiegt und Unlustgcfühle so ıur dann zum Bewußtsein kommen läßt, weın das Lustgefühl bei der eigenen Aktivität fchlt.

Weniger bedeutungsvoll, aber oft recht störend ist die Uebcrempfindlichkeit gegen Gleichgewichtsstörungen, die beim Karusselfahren, Schaukehl, Eisenbahn-, Wagenfahren der Kinder zu Unlustgefühlen einfacher Art, aber auch zu Uebelkcit oder gar reflektorisch zu Erbreehen führt. Gerade diese Störung tritt exquisit fanniliär, oft zienlich isoliert auf und pflegt im späteren Kindesalter, manehmal auch erst nach der Pubertït zu verschwinden.

Sehr charakteristisch ist schließlich die Ueberempfindlichkeit gegen internittierende $\mathrm{Hautreize}$, die die Kinder überempfindlich macht gcgen dic juckenden und kratzendeı Gefülıle der wolleneı Strünpfe, der Ränder der Beinkleider oder Aermel, gegen die Bcrührung von Samt, gegen das Nägelschneiden und ähnliches. Diese Störungen nehınen oft einen hohen, ja beängstigenden Grad an und gehen dann unmittelbar über in Zwangszustände, die eruster Beliandlung bcdürfen. Diese Störung ist für das Kindesalter bis zu gewissem Grade pathognonomiseh und nicht sehr ernst zu nchmen, da ja den Tastempfindungen überhaupt für das frühkindliche Alter eine besonders große Bedeutung zukommt.

Auch die allgemeine Uebercmpfindlichkcit gegen Sch m er dürfte an dieser Stelle einzurangieren sein.

c) Eine noch größcre Rolle als in Gruppe b spielt die Mitbeteiligung des psychischen Faktors bei einer dritten Gruppe, die wir aber zu den neuropathischen Störungen noeh zuzurechnen doch wohl berechtigt sind. Es sind das dicjenigen Störungen, die mit der Bildung und Lösung der zeitlichen individuellen, bedingten Reflexe im Zusammenhange und die dadwrch zu den Störungen der bisher beschriebenen , unbedingten" Reflexe in gewissem Gegensatz stehen. Der Untersehied liegt vor allenl darin, da B die vorher in Betracht gezogenen Reflexe bei allen Menschen in Prinzip in gleichen Bahnen verlaufen, während bei den bedingten Reflexcn nur für gewisse Zeit und für ein bestimmtes Individuum sich dic Verknüpfungen zwischen Reiz und Erfolgsreaktion' bilden. Auf die ganze Theorie der bedingten Reflexe, wie sie von dem Russen Pawlow, Bechterew, Krasnogorski geschaffen, von Czerny ${ }^{1}$ ) und $\mathrm{Ibrahim}^{2}$ ) für die Kinderheilkunde nutzbar gemacht worden ist, einzugehen, ist hier nicht der Ort. Wiehtig für die hier in Betracht kommenden Störungen dürfte bcsonders die Erfahrung von Krasnogorski ${ }^{3}$ ) sein, daß bci neuropathischen Kindern die bedingten Reflexe sich manchmal leichter bilden, immer aber schwcrer lösen lassen als bei nicht neuropathischen Kindern. Diese Erfahrung aber ist geeignet, uns das Verständnis für die Tatsache zu erleichtern, da $B$ sich gewisse Angewohnheiten bei neuropathisehen Kindern leichter fixieren und schwerer beseitigen lassen als bei anderen Kinderı.

Die genannte Erfahrung spielt einmal eine Rolle bei denjenigen Gewohluheiten, die wir im Säuglingsalter noch nicht als patlologiseh ansehen dürfen, dcren Festhaften bis in das spätere Kindesalter aber die Grenze des Physiologischen überschreitet. Dahin gehören das Da u m en lu tsch en und wohl auch die Jactatio eapitis nocturna. Es kann sicher nicht als krankhaft angesehen werden, wenn ein Säugling reflektorisch an Gegenständen, die ilım in den Mund kommen, z. B. den eigenen Fingerchen, lutscht und wenn sich damit Lustgefühle und Beruhigung verbinden. Bleibt diese Gewohnheit aber bis in das 4. Jahr bei Tage bestehen, oder ist gar die Fähigkeit, überhaupt einzuschlafen, bei einem 10 jährigen abhängig von den Lutschen am Finger, so müssen wir darin, besonders wenn viele vergebliche Versuche zur Abgewöhnung gemacht sind, eine pathologische Perseveration erblicken. In noch höherem Grade gilt das Gleiche, wenn ein Säugling, der bei

1) Stralbburger m. Ztg. 1910 H. 9. - 2) Zschr. f. d. ges. Neurol. u. Psych. 5. 1911. H. 3 u. Neurol. Zbl. $1911 \mathrm{Nr}$. 13. $-{ }^{3}$ ) Jb. f. Kindhlle 78. 1913. H. 4. passivem Geschaukeltwerden in normaler Weise Lustgefühle empfindet, zufällig erlernt, durch aktives Schaukeln des Kopfes oder der Arme sich diese Lustgcfühle selbst zu verschaffen. Eine solche Gewohnheit kaun nicht ohne weiteres als krankhaft angesehen werden; nimmt sie jedoch einen so hohen Grad an, daB das Kind die ganze Nacht, Wochen und Jahre hindurch, den Kopf sehaukelt, sodaB dic Haare am Hinterkopf ganz fortgescheuert sind, dann haben wir zweifellos eine pathologische Gewohnheit vor uns, die in einem großen 'Teil der Fälle - doch kommit auch Schwachsinn in Betracht - als neuropathisehes Zeichen angesehen werden mu B.

In anderen Füllen nü̈ssen wir als Ursache des Beginns der häßlichen Angewohnheiten, also der bedingten Reflexc, eine Uebererregbarkeit der Hautsinucsgebiete ansprechen, die die Kinder veranla Bt, Wachstums- und Hautreize, die von anderen kaunı als solche empfunden werden, dureh Gegenreize zu bcseitigen und diese dadurch entstehenden Lustgefühle immer von neuem aufzusuchen. Hicrher gehören das Haarausreißen, Nasebolırcn, das Abreißen der Haut neben den Fingernägeln und vor allem das Nägclkauen, das einer crzieherischen Bceinflussung ganz bosonderen Widerstand entgegensetzt.

Leichter zu beseitigen dagegen ist die Fixicrung derjenigen bedingten Reflexe, die urspriinglich aus cincr rein reflektorischen $\mathrm{Ab}$ wehrbew egung cntstanden sind, den Charakter der einfachen oder ausgedehnteren Reflexzuckung beibehalten habel1, also der bekannten Tics, die cigentlich den Urtypus der ,.läßlichen Angewohnheitcn" darstellen und aus den beiden Wurzeln der Uebererregbarkeit einerseits und der Erschwerung der Lösung bedingter Reflexe anderscits sich ableiten lassen. Der Uebergang von hier zu den eeliten Phobien und Zwangsvorstellungen, der uns schon zu komplizicrteren scelischen Störungen linüberfühırt, ist, wie bckannt, fließcnd.

Am kompliziertesten liegen dic Verhältnisse, wenn häßliehc Angewolnhleiten sich entwickeln in Zusammenhang mit ciner gesteigerten Erregbarkeit auf dem Gebietc des vegetativen Nervensystems, wie wir es bei den,,respiratorischen Affekt. krämpfen" (Ibrahim) und bei der Neigung niancher Kinder sehen, auf dell Aublick bestimmter Gegenstände (z. B. Blut) oder auf sonstige Reizc bestiminter Art nit $\mathrm{Ohn}$ machten zu rcagieren. In letzter Linie sind aber auch das bedingte Reflexe zusammengesetzter Art, die bei neuropathischen Kindern sich bilden und in pathologischer Weise fixieren, dementsprechend aber auch durch erzieherischc Einflüsse durchbrochen und beseitigt werden können.

Zusammenfassung. Als neuropathisch zu bezeichnen sind diejenigen Kinder, bei denen in intensiver oder gehäufter Fornı auf eincr fchlerhaften Anlage beruhende Funktionsstörungen der phylogenetisch ältesten Teile des nervösen Apparates sich zeigen, die als Steigerung der reflektorischen Erregbarkeit des zentralen oder vegetativen Nervensystems angesprochen werden müssen. Dic so entstehenden Störungen manifestiercn sich entweder im Gebiet der unbedingten Reflexe als erleichtcrte, verbreiterte oder abnorm intensive motorische Reaktionen auf leichte bzw. mittelschwere Rcize, oder als ein verfrülites Auftreten von Unlustgcfühlen bei den gleichen Reizen, oder, in Gebiet der bedingten Reflexe, als abnorin langes und intensives Festhaften sogenannter läßlicher Angewohnliciten. 